\title{
Effects of shear velocity oscillations on stick-slip behavior in laboratory experiments
}

\author{
Heather M. Savage ${ }^{1}$ and Chris Marone ${ }^{1}$ \\ Received 21 December 2005; revised 15 September 2006; accepted 22 September 2006; published 15 February 2007.
}

[1] We report on laboratory friction experiments in which simulated faults are exposed to shear velocity oscillations at different amplitudes and frequencies. Granular layers are sheared in a servo-controlled biaxial apparatus at constant normal stress and background shear velocity, with a shear velocity sinusoid superimposed. Correlation between oscillations and stick-slip events is determined by the timing of dynamic failure with respect to the oscillation phase. Schuster's test is used to calculate the statistical likelihood of phase recurrence. We find that correlation of failure with the shear stress oscillation depends on oscillation frequency and amplitude at low frequencies and solely on oscillation amplitude at high frequencies. The frequency boundary between these two regimes is proportional to the inverse of the time needed to displace the frictional critical slip distance. We evaluate changes in failure characteristics, including failure strength, recurrence time between events, creep, and phase of the oscillation at failure, to assess the effects of stressing rate oscillations. Failure occurs at maximum shear stressing rate at low frequencies and lags peak stressing rate in the high-frequency regime. Friction at the onset of dynamic failure decreases with increasing frequency. The distribution of events through time depends on the frequency of the shear oscillation; low-frequency oscillations produce bimodal distributions and high-frequency oscillations produce unimodal distributions. If the transition between the failure regimes depends on the critical displacement length as our experiments imply, the critical frequency will vary for faults with different gouge layer thicknesses and total displacement.

Citation: Savage, H. M., and C. Marone (2007), Effects of shear velocity oscillations on stick-slip behavior in laboratory experiments, J. Geophys. Res., 112, B02301, doi:10.1029/2005JB004238.

\section{Introduction}

[2] Fault zones experience periodic deviations in their stress state at various magnitudes and timescales. These range from long timescale static changes, such as tectonic loading due to interaction with a nearby fault, to transient high-frequency changes such as seismic waves or solid Earth tides [Cochran et al., 2004]. Whereas static stress transfer alters the fault boundary conditions, transient stresses can change material response of the fault zone but do not permanently alter the stress state. Both static and dynamic stress variations induce changes in seismicity rates; however, only transient stresses can trigger remote earthquakes (greater than a few fault lengths from the source). The effects of static stress transfer have been relatively well studied and are considered in earthquake hazard assessments. In contrast, the effects of transient stress transfer are poorly understood. For example, not all large earthquakes trigger remote seismicity and certain geographic regions seem more susceptible to triggering [Gomberg et al., 1997; Brodsky et al., 2003; Gomberg et al., 2004; Brodsky and

\footnotetext{
${ }^{1}$ Rock Mechanics Laboratory, Department of Geosciences, Pennsylvania State University, University Park, Pennsylvania, USA.

Copyright 2007 by the American Geophysical Union. 0148-0227/07/2005JB004238\$09.00
}

Prejean, 2005]. When stresses are periodic, high-frequency oscillations require larger stress amplitudes to induce seismicity than static stress changes [Beeler and Lockner, 2003]. Understanding transient stress effects on fault strength and stability would enhance our knowledge of how earthquakes are triggered.

[3] Dynamic triggering in natural earthquake settings does not seem to depend on the magnitude of the triggering earthquake nor distance from the event, but could be a function of the oscillating stress frequency [Brodsky and Prejean, 2005] or amplitude [Gomberg and Johnson, 2005; Johnson and Jia, 2005; Felzer and Brodsky, 2006]. Furthermore, the time delay between triggering and the triggered event varies. Triggered earthquakes may be events that were inevitable, such that dynamic triggering merely sped along their occurrence [Gomberg et al., 1997]. Additionally, the time delay between trigger and triggered event may depend on how close the fault was to failure. Simple models for fault zone failure, such as the Coulomb-Amonton failure law, predict that failure occurs instantaneously when the shear stress resolved on the fault exceeds the fault strength, which is independent of rate or time. Therefore time/stress path dependence of failure due to dynamic stressing is not explained by such models and requires a more complicated context. 
[4] Recent studies have shown that large tidal variations can trigger earthquakes. However, both location and type of event seem to dictate whether high or low tides trigger instabilities. For example, Cochran et al. [2004] found a correlation between high tides and thrust earthquakes around the Pacific Rim, especially in areas with larger high tides (and consequently larger loading amplitudes). In contrast, microseismicity on the Juan de Fuca Ridge peaks with low tide [Tolstoy et al., 2002]. Studies of earthquakes in Antarctic glacial till have found that tidally modulated driving stress at the end of the ice sheet may induce a daily displacement pattern, that is analogous to episodic slipping on a locked fault. The largest tides support the end of the ice sheet and thereby relieve load, such that the lowest shear stress applied to the till layer occurs during high tide. Unstable slip of the ice sheet tends to occur during falling tides, when shear stress on the till layer is increasing [Bindschadler et al., 2003]. Studies of deep moonquakes also suggest a strong tidal influence [Lammlein, 1977].

[5] Longer period stress fluctuations may also trigger seismicity. Seasonal groundwater recharge creates a peak in seismicity lagging the spring snowmelt at Mt. Hood by 151 days [Saar and Manga, 2003]. The increase in compression associated with snow load may also induce seismicity. Heki [2003] found an increase in magnitude $\geq 7.0$ earthquakes in spring and summer months in regions where there is a winter snowpack that did not exist in regions without significant snowpack.

[6] Past laboratory studies of stress vibrations have focused on normal stress perturbations [Richardson and Marone, 1999; Boettcher and Marone, 2004; Hong and Marone, 2005] or oscillations of principal stresses [Beeler and Lockner, 2003]. Normal stress vibrations experienced by a locked fault can cause frictional relaxation during vibrations and greatly increase healing effects after vibrations cease [Richardson and Marone, 1999]. Other studies have shown that high-frequency, large-amplitude vibrations reduce the shear strength of a creeping fault and that intermediate frequencies induce a lag between normal and shear stress peaks, leading to fault destabilization [Boettcher and Marone, 2004]. However, additional work has shown that normal load modulation on a stick-slipping fault can lead to stabilization [Cochard et al., 2003]. Beeler and Lockner [2003] tested the effect of shear and normal stress oscillations on bare granite surfaces and found that two different shear stress responses occurred, depending upon whether the frequency of the oscillation was greater or less than the nucleation time of a stick-slip event. In the low-frequency regime, the timing of instability was influenced by the wave period. Failure in the high-frequency regime depended on the amplitude of the oscillations but not the period.

[7] We analyzed shear stress response of a laboratory fault to periodic shear loading rate oscillations. Our experiments are similar to those reported by Lockner and Beeler [1999] and Beeler and Lockner [2003]; however, we oscillate shear loading rate and maintain a constant normal stress. Additionally, our experiments are conducted on a granular material. A constant shear loading velocity, representing remote stress, was summed with a sinusoidal wave simulating a transient oscillation. We deciphered how these oscillations influenced the material response by varying loading rate, as well as amplitude and frequency of the oscillations. Our laboratory faults experienced stick-slip instabilities analogous to the earthquake cycle both during shear vibrations and under nonoscillating load conditions. We measured changes in the stick-slip cycle such as recurrence interval, failure strength, phase lag and the preseismic and coseismic slip to determine how shear oscillations may affect fault stability. Changes in failure strength would eliminate the possibility that instabilities are due to a simple Coulomb-style failure where instability occurs at a constant stress threshold. We also measure recurrence interval, phase lag and slip because these may be measurable quantities on natural faults that could point us to potential examples of earthquake triggering.

\section{Experimental Technique}

[8] Experiments were conducted using a servo-controlled, biaxial apparatus with a double-direct shear configuration (Figure 1 inset). The samples were loaded in shear displacement servo-controlled feedback with $0.1 \mu \mathrm{m}$ resolution to achieve a constant displacement rate of a load point at the top of the center block of the double-direct shear assembly (Figure 1 inset). The load point displacement history consisted of a linear function with a sinusoid superimposed to mimic a tectonic load and oscillating stress. A constant normal stress of $5 \mathrm{MPa}$ was implemented with a servocontrolled load-feedback mechanism (with $0.1 \mathrm{kN}$ resolution); such low normal stresses eliminate comminution of the sample and any associated changes in stick-slip behavior. The stiffness of the vertical load frame is $5 \mathrm{MN} / \mathrm{cm}$ or $250 \mathrm{MPa} / \mathrm{cm}$ when expressed as the shear stress on a double direct shear sample with nominal friction contact dimensions of $10 \mathrm{~cm} \times 10 \mathrm{~cm}$. Stiffness of the apparatus and sample assembly is approximately $0.017 \mathrm{MPa} / \mu \mathrm{m}$, as determined in situ by measuring shear stress-displacement curves.

[9] We sheared 3-mm-thick layers of soda lime glass beads (size distribution 105-149 $\mu \mathrm{m}$, Mo-Sci Corporation, Rolla, Missouri) at room temperature and humidity. Humidity has been shown to affect the shear strength and recurrence interval of laboratory faults [Frye and Marone, 2002], so we do not make interexperiment comparisons of strength and recurrence interval. Instead, we only discuss the absolute value of changes in recurrence and failure strength within one experiment, but demonstrate how patterns in the changes with frequency are similar between experiments. Glass beads have material properties similar to quartz and are an ideal substance for these tests due to the repeatability of the magnitude and recurrence interval of stick-slip events. The samples were sheared between steel forcing blocks of size $10 \mathrm{~cm} \times 10 \mathrm{~cm} \times 2 \mathrm{~cm}$ (side blocks) and $10 \mathrm{~cm} \times 15 \mathrm{~cm} \times 3 \mathrm{~cm}$ (center block) so that a constant nominal contact area of $10 \mathrm{~cm} \times 10 \mathrm{~cm}$ was maintained throughout shear. On the surfaces that are in contact with the sample, the steel blocks have triangular grooves $0.8 \mathrm{~mm}$ deep and $1 \mathrm{~mm}$ in wavelength cut perpendicular to the shear direction. These grooves force shear to occur within the sample layer instead of along the boundary. Steel guide plates and tape were attached to the unconfined sides of the sample configuration to hold the blocks together until loaded. Copper shims were attached at the bottom of the sample layers to minimize gouge loss. A latex rubber sheet 


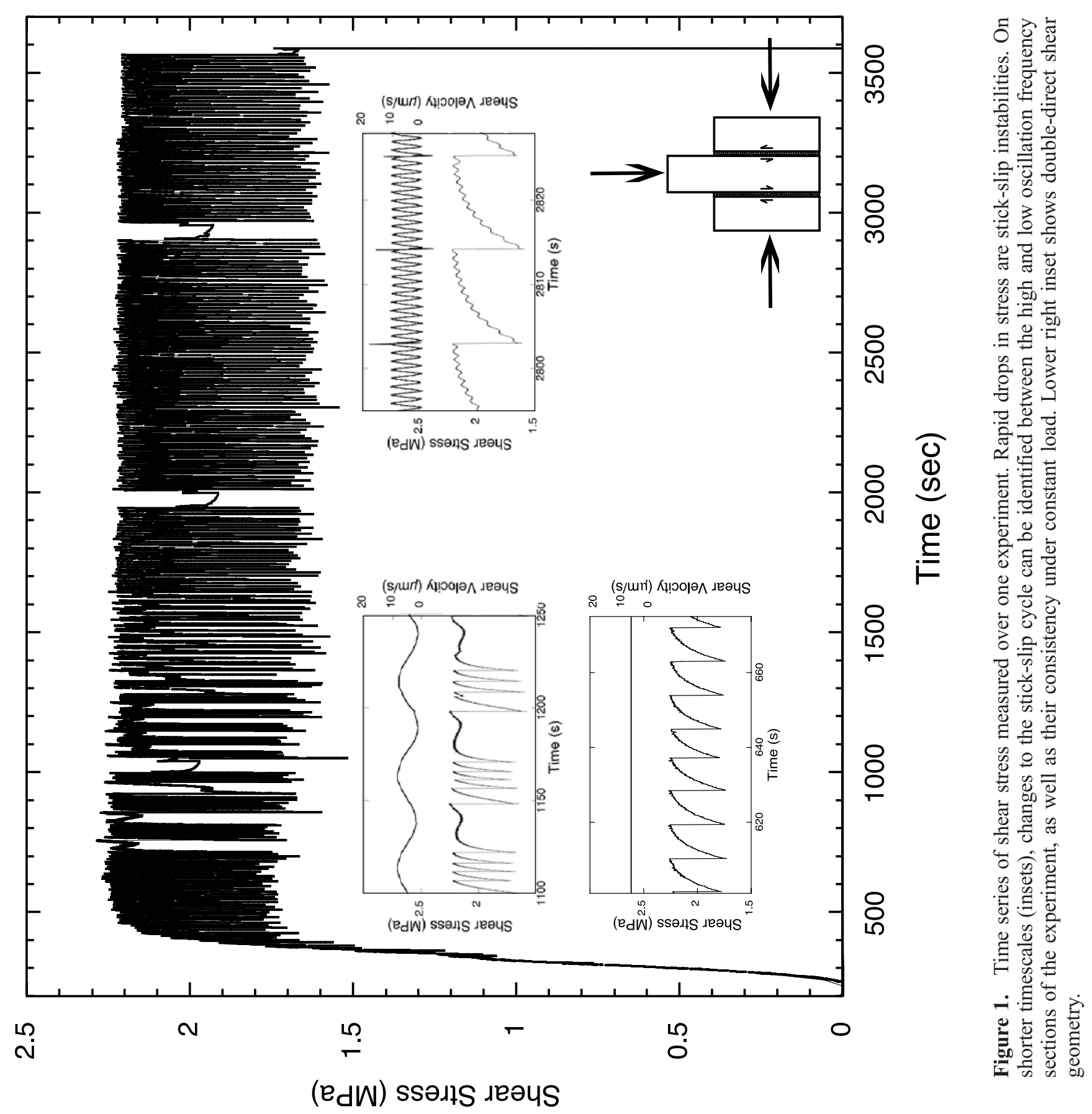


Table 1. List of Experiments

\begin{tabular}{cccc}
\hline Experiment & $\begin{array}{c}\text { Background } \\
\text { Loading Rate, } \mathrm{MPa} / \mathrm{s}\end{array}$ & $\begin{array}{c}\text { Frequency, } \\
\mathrm{Hz}\end{array}$ & $\begin{array}{c}\text { Amplitude, } \\
\mathrm{MPa} / \mathrm{s}\end{array}$ \\
\hline p269 & 0.17 & 1 & 0.085 \\
p337 & 0.17 & 1 & 0.17 \\
p338 & 0.17 & 0.1 & 0.17 \\
p346 & 0.17 & 0.5 & 0.17 \\
p393 & 0.17 & 0.5 & 0.085 \\
p505 & $0.85-0.17$ & $0.02-1$ & $0.051-0.17$ \\
p506 & 0.34 & $0.1-2$ & $0.085-0.34$ \\
p518 & 0.17 & $1-2$ & $0.034-0.17$ \\
p586 & $0.085-0.34$ & $0-3$ & $0-0.34$ \\
p589 & $0.085-0.17$ & $0.1-0.5$ & 0.051 \\
p591 & 0.085 & $0.5-1$ & $0.017-0.051$ \\
p595 & 0.085 & 0.2 & $0-3$ \\
p598 & 0.34 & $0-3$ & $0-0.34$ \\
p600 & 0.085 & $0.5-1$ & $0.017-0.051$ \\
p605 & 0.34 & $0.01-3$ & 0.34 \\
p620 & 0.085 & $0.01-2$ & 0.085 \\
p648 & 0.17 & $0.01-2$ & 0.17 \\
p707 & $0.17-0.34$ & $0.01-3$ & $0.085-0.34$ \\
p708 & 0.17 & $0-3$ & $0-0.17$ \\
p997 & 0.34 & $0.02-4$ & $0.051-0.17$ \\
p1005 & $0.085-0.17$ & $0.02-4$ & $0.017-0.085$ \\
\hline
\end{tabular}

of $0.01^{\prime \prime}$ thickness was taped over the copper shims, also to reduce gouge loss.

[10] The positions of both rams were measured by displacement transducers (DCDTs) throughout each experiment. Using the position of the vertical ram and correcting for the stiffness of the loading apparatus allowed us to determine shear displacement at the gouge layer boundaries and shear strain within the layer. The amount of preseismic and coseismic slip was recorded at each instability. Recording the displacement of the horizontal ram throughout the experiment allowed for reconstruction of the change in gouge thickness both over the course of the entire experiment due to geometric thinning, as well as for dilation and compaction of the sample during the stick-slip cycle.

[11] The amplitude and frequency of the oscillation, as well as background loading rate, were varied to study the material response (Table 1). Three background loading velocities were used: 5,10 , and $20 \mu \mathrm{m} / \mathrm{s}$, which correspond to shear stressing rate oscillations of $0.085,0.17$, and $0.34 \mathrm{MPa} / \mathrm{s}$, respectively (given a stiffness of $0.017 \mathrm{MPa} / \mu \mathrm{m}$ ). Loading rate oscillation amplitudes ranged from 1 to $20 \mu \mathrm{m} / \mathrm{s}$, depending on the background loading rate. In the figures herein, we present oscillation amplitudes in terms of shear stress amplitude, determined by integrating over one quarter of the stressing rate oscillation period. We varied mean loading rate and amplitude such that the effective loading rate was always positive and finite shear load was maintained on the sample throughout the experiment. Frequencies ranged from $0.01 \mathrm{~Hz}$ to $4 \mathrm{~Hz}$. At frequencies above $4 \mathrm{~Hz}$ and amplitudes smaller than $1 \mu \mathrm{m} / \mathrm{s}$ our signal-tonoise ratio was too small to allow accurate measurements of the material response; thus this was our lower limit of amplitude and upper limit of frequencies.

[12] The sample was preconditioned at a constant shear velocity for approximately $1 \mathrm{~mm}$ of displacement until a steady state maximum shear stress was reached (Figure 1). Because the glass beads behaved unstably throughout shearing, we used the maximum shear stress to assess whether steady state strength had been reached. After achieving steady state conditions, the sinusoid was added to the computer controlled displacement signal (Figure 2a). The sinusoid signal remained at a constant amplitude and frequency for
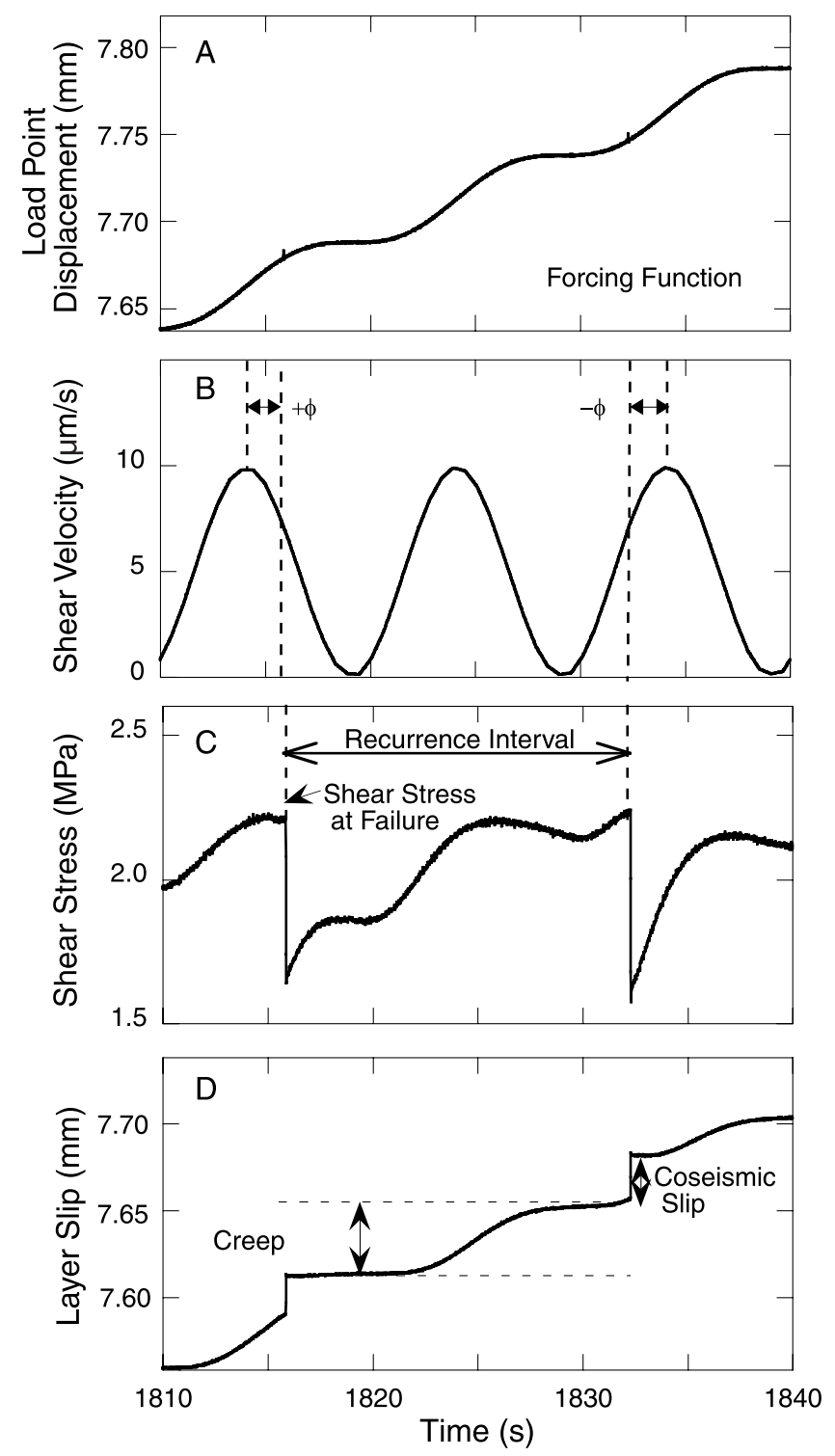

Figure 2. Illustration of key experimental parameters and boundary conditions for loading. (a) A constant load point displacement of $5 \mu \mathrm{m} / \mathrm{s}$ (shear stress rate of $0.085 \mathrm{MPa} / \mathrm{s}$ ) was prescribed and an oscillation of $0.085 \mathrm{MPa} / \mathrm{s}$ was superimposed. (b) Phase lag is defined as the difference between the peak in the shear velocity curve (zero phase) and the oscillation phase at the time of failure. Instabilities occurring after the peak have a positive phase shift whereas failure prior to the peak is defined as a negative shift. (c) Shear stress at failure is the shear stress value at the onset of dynamic failure and recurrence interval is the time between successive stick-slip events. (d) The creep that occurs between stick slips is referred to as preseismic slip and the displacement that occurs during dynamic failure is called coseismic slip. 
A

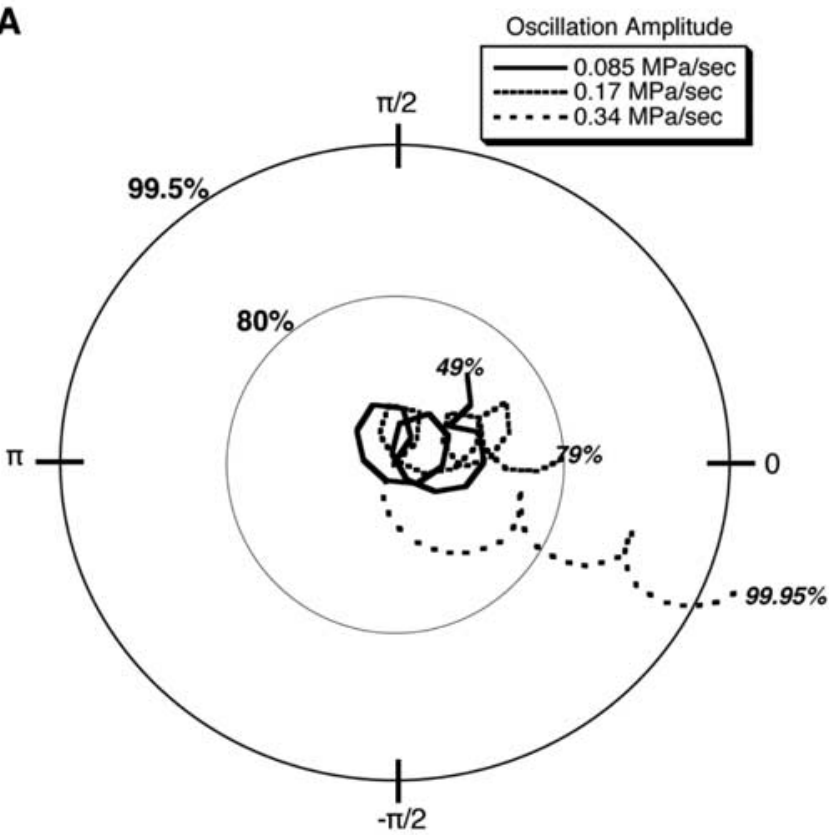

B
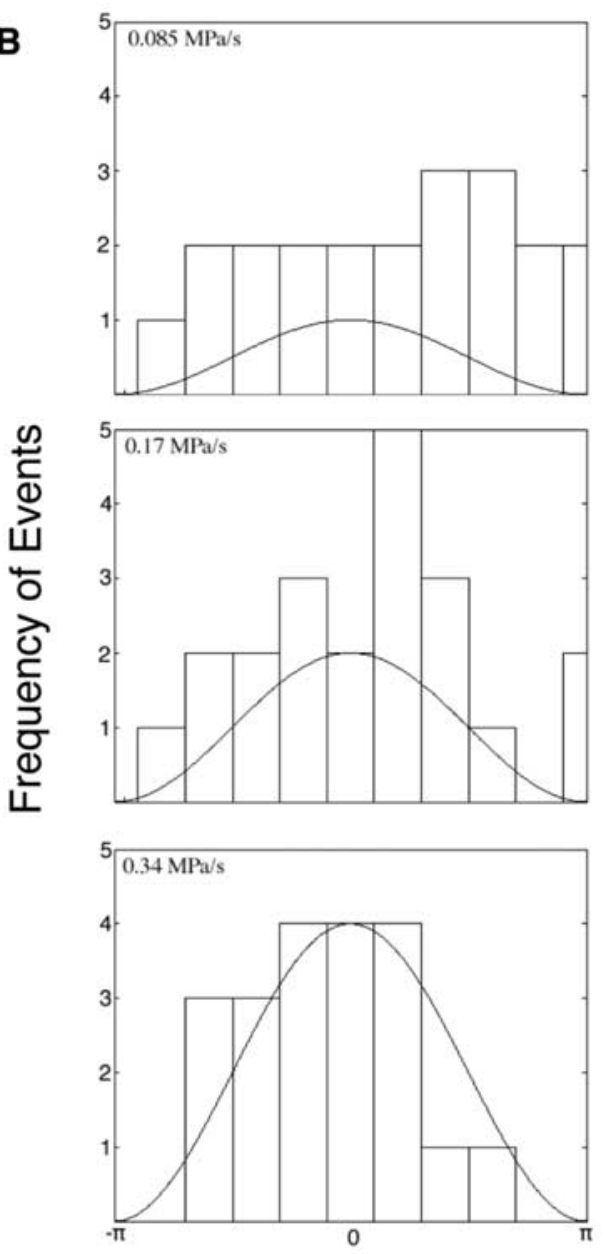

Phase

Figure 3. (a) Correlation between instabilities and loading rate oscillations for experiments with a constant frequency of $0.05 \mathrm{~Hz}$ was determined using Schuster's test. Correlated series walked out a distance greater than the radius of the $99.5 \%$ probability circle (the experiment with a $0.34 \mathrm{MPa} / \mathrm{s} \mathrm{shear}$ loading rate oscillation amplitude), while uncorrelated series did not walk far from the origin. The scalloped pattern of the walk (seen most clearly in the $0.34 \mathrm{MPa} / \mathrm{s}$ data) is characteristic of low-frequency experiments (where the wavelength of the "scallop" is determined by the period of the stressing rate). (b) The histograms demonstrate the relationship between stick-slip frequency and loading rate (sinusoid) for the data shown in Figure 3a.

$2 \mathrm{~mm}$ of displacement, which was the approximate displacement needed to induce more than 20 stick-slip instabilities.

\section{Results}

[13] The timing of the shear stress response, i.e., stick-slip instabilities, was measured in terms of the phase angle relative to the stressing rate sinusoid (Figures $2 \mathrm{~b}$ and $2 \mathrm{c}$ ). We define the peak in stressing rate as the zero phase of the oscillation. The phase angles at each failure time are tested for statistical similarity, which we call a correlation with the shear oscillation. Correlation between the oscillations and the stick-slip events was measured using a Schuster, or random walk test [Rydelek and Hass, 1994]. We test the probability that a series of events occurred nonrandomly by treating each event as a step of unit length. Starting at the origin of a circle, each step is placed head to tail at the phase angle in which the event occurred in the oscillation (Figure 3a). If each event occurred at a similar angle, the distance from the origin to the end of the walk, D, will be statistically significant. If the series of instabilities occur at random phases of the oscillation, the walk will end near the origin. The probability that a distance is random is described by:

$$
P=\exp \left(\frac{-D^{2}}{N}\right)
$$

where $\mathrm{N}$ is the number of events in the data set. We express nonrandomness as a correlation percent, $(1-\mathrm{P}) * 100 \%$. Values above $99.5 \%$ correlation are deemed strongly correlated. Figure $3 \mathrm{~b}$ shows the same data set as Figure $3 \mathrm{a}$ as histograms to illustrate how events are more distributed over the entire sinusoid when events are uncorrelated. 

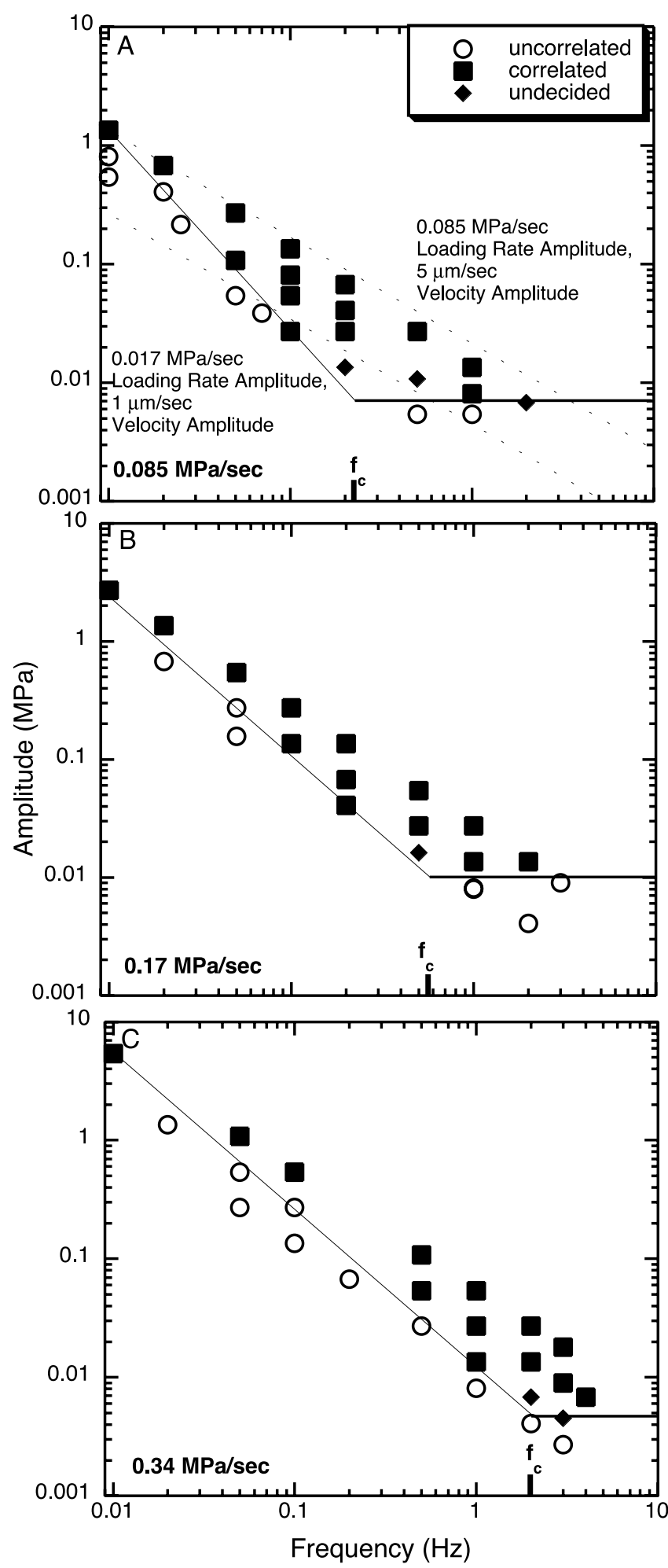

Figure 4. Correlation thresholds for three loading rates. At low frequencies, increasing the background loading rate required larger shear amplitudes to produce consistent correlation. Generally, doubling the background loading rate required a $\sim 2.5$-fold change in amplitude to achieve correlation. At high frequencies, the threshold boundary did not vary systematically with stressing rate amplitude. The minimum shear amplitude needed for correlation at high frequencies varied between $\sim 0.04$ and $0.1 \mathrm{MPa}$. The critical frequency, $\mathrm{f}_{\mathrm{c}}$, is positively correlated with shear loading rate.
[14] We find that correlation between stress oscillation and instability depends on both the amplitude and frequency of the oscillations (Figure 4). Oscillation amplitudes are presented here as shear stress values for comparison to similar studies of the correlation threshold [Lockner and Beeler, 1999; Beeler and Lockner, 2003]. The prescribed loading rate oscillation was recalculated as a shear stress oscillation by using the system stiffness (apparatus plus the sample; $0.017 \mathrm{MPa} / \mu \mathrm{m}$ ) to compute a stressing rate and then integrating the stressing rate to obtain a shear stress. For example, a $1 \mathrm{~Hz}$ oscillation of velocity amplitude $10 \mu \mathrm{m} / \mathrm{s}$ is equivalent to a $0.17 \mathrm{MPa} / \mathrm{s}$ stressing rate amplitude and a $0.027 \mathrm{MPa}$ stress amplitude. Points that experienced the same stressing rate amplitude lie on lines of constant slope (see dashed lines in Figure 4a). The correlation boundary was well constrained for most of the data. Some points varied around the $99.5 \%$ threshold and are called "undecided" points (diamonds in Figure 4).

\subsection{Correlation Threshold}

[15] We found that the correlation threshold exhibits two frequency regimes with respect to the stress amplitude perturbation required to produce correlation (Figure 4). In the low-frequency regime, the correlation threshold is frequency-dependent, and has a negative slope $(-0.07$, -0.22 and $-0.37 \mathrm{MPa} s$ for background stressing rates of $0.085,0.17$ and $0.34 \mathrm{MPa} / \mathrm{s}$, respectively), such that

$$
\log A=m \log \omega
$$

where $\mathrm{A}$ is amplitude and $\omega$ is frequency. The boundaries separating correlated and uncorrelated conditions in Figure 4 were determined by eye. In most cases, the placement of the thresholds was well constrained (Figure 4); however, for our largest background stressing rate (Figure 4c) the (apparent) transition occurs near our upper limit of well constrained oscillations, and therefore is less well determined.

[16] The stress perturbation needed to overcome the correlation threshold is larger at lower frequencies and decreases toward higher frequencies before leveling off in a high-frequency regime. For high frequencies, we find that changes in stress must exceed a minimum amplitude in order to induce a correlation between stick-slip instability and loading rate oscillation and that the correlation threshold is frequency-independent (Figure 4). Both of these correlation regimes were observed for our two slower loading rates (Figures $4 \mathrm{a}$ and $4 \mathrm{~b}$ ); however, the high-frequency threshold is not well constrained at the fastest background loading rate (Figure 4c). At low frequencies, the amplitude of the correlation threshold was directly proportional to the background loading rate. This means that for higher loading rates, a larger stress amplitude was needed to achieve correlation conditions. However, the correlation threshold in the high-frequency regime did not change systematically with loading rate (Figure 4). This may mean that the threshold is constant (approximately $0.01 \mathrm{MPa}$ ) and/or that it varies at a level below the signal-to-noise ratio in our experiments.

[17] The crossover time, or inversely the critical frequency $\mathrm{f}_{\mathrm{c}}$, is the frequency at which the correlation threshold changes from frequency-dependent to frequency-independent. We determined $f_{c}$ by estimating where these thresholds inter- 
sected in Figure $4(0.22,0.55$, and $2 \mathrm{~Hz}$ for $0.085,0.17$, and $0.34 \mathrm{MPa} / \mathrm{s}$ stressing rates, respectively).

[18] The correlated points in Figure 4 represent instabilities that have been triggered by the stress oscillations. In the following sections, we look for changes in the seismicity pattern caused by the frequency and amplitude of the oscillations at the correlated points.

\subsection{Recurrence Interval}

[19] In correlated experiments shown in Figure 4, the timing of stick slips occurs at a specific phase angle, thereby altering the natural recurrence interval between stick-slip events compared to a nonoscillating loading rate. Recurrence interval was measured from the beginning of an instability to the beginning of the next instability (Figure 2). The recurrence interval for a nonoscillating load was calculated and averaged for each background loading rate and expressed as a frequency $\left(\mathrm{f}_{0}\right)$. The value of $\mathrm{f}_{0}$ varied slightly between experiments conducted at the same loading rate (see value of $\mathrm{f}_{0}$ between (Figures 5 (left) and 5 (right)). We find an evolution of the recurrence interval pattern from low to high frequencies. For frequencies below the threshold $\mathrm{f}_{0}$ recurrence interval is bimodal whereas above $\mathrm{f}_{0}$, stick-slip exhibits a unimodal recurrence interval. This pattern can be seen for all three background loading rates (Figure 5).

[20] In the low-frequency regime, the bimodal distribution of recurrence intervals is caused by the clustering of instabilities around loading rate peaks (Figure 6). Most of the instabilities are close together in time and occur at or near the loading rate peak. The second group of longer recurrence intervals is the result of shear velocity falling to zero in the trough of the stressing rate oscillation, which causes instabilities to cease. After velocity begins to increase, instabilities resume. The mean of this group of longer recurrence intervals generally decreases as the vibration frequency decreases (Figure 5), until the vibration frequency becomes higher than $f_{0}$. The recurrence interval stays generally constant under high-frequency oscillations (Figure 5); however, they must be slightly perturbed in order to correlate with the oscillation.

\subsection{Instability Phase}

[21] We calculate the mean phase shift of dynamic failure from the maximum stressing rate for correlated points (Figure 2). The lower-frequency oscillations (below $\mathrm{f}_{\mathrm{c}}$ ) produce stick slips with an average phase of zero, with a possible trend toward negative numbers at frequencies lower than $\mathrm{f}_{0}$ (Figure 7). The most negative phase shift coincides with oscillation frequencies just below $\mathrm{f}_{0}$ $(0.05 \mathrm{~Hz}, 0.1 \mathrm{~Hz}$, and $1 \mathrm{~Hz}$ for background loading rates $0.085 \mathrm{MPa} / \mathrm{s}, 0.17 \mathrm{MPa} / \mathrm{s}$, and $0.34 \mathrm{MPa} / \mathrm{s}$, respectively). The higher-frequency oscillations (above $f_{c}$ ) force stick slips to occur after the stressing rate peak (between around $33^{\circ}-44^{\circ}$ ) for all background stressing rates. Oscillation amplitude (when frequency is held constant) does not produce a consistent phase shift. For instance, at $2 \mathrm{~Hz}$, the smallest amplitude oscillations create the largest phase shift, the intermediate amplitude creates the smallest phase shift and the largest amplitude creates an intermediate phase shift (Figure 7c).

\subsection{Friction at Failure}

[22] For experiments in which stick slip was correlated with stressing rate oscillations, the maximum friction value at dynamic failure varies as a function of the amplitude and frequency of the shear stressing rate oscillation (Figure 8). Frictional failure strength decreases with increasing frequency below $f_{0}$ and is relatively constant above $f_{0}$. The amount of weakening is greatest at the fastest background loading rate (Figure 8c); however, weakening is not linearly dependent on loading rate. Slight deviations from the weakening rate include an increase in the weakening effect at frequencies just below $\mathrm{f}_{0}$ (at $0.02 \mathrm{~Hz}, 0.05 \mathrm{~Hz}$ and $0.1 \mathrm{~Hz}$ for background loading rates $0.085,0.17$, and $0.34 \mathrm{MPa} / \mathrm{s}$, respectively) and a strengthening effect at frequencies higher than $f_{0}$ and at or near the critical frequency $\left(f_{c}\right)$. The weakening effect loosely coincides with the frequency that exhibits the anomalously shorter recurrence interval (Figure 5).

\subsection{Preseismic and Coseismic Slip}

[23] We refer to the inelastic creep that occurs during the dilational phase of the stick-slip cycle and prior to failure as preseismic slip (Figure 2d). The pattern of preseismic slip follows that for coseismic (Figure 9). The fastest background loading rate produces the most preseismic slip; however, preseismic clip varies only slightly at the lower background loading rates. Like the recurrence interval pattern, preseismic slip varies widely at the lower frequencies. Recurrence interval and preseismic slip covary because as time between events increases, so will the length of time the layer is creeping. The failure strength, as well, is negatively correlated with slip. Where preseismic slip is greater, the failure strength decreases (as wee see in frequencies less than $f_{0}$ ) because the failure strength depends on age of grain contacts.

\section{Discussion}

\subsection{Correlation Threshold}

[24] We find that stick-slip instabilities correlate with shear loading rate oscillations, possibly in two regimes; one regime in which the correlation is determined by both the amplitude and frequency of the oscillation and a second where only the amplitude of the oscillation affects correlation. Simple triggering by static stress changes (such as the Coulomb failure model) cannot explain the two regimes. We plot the correlation boundary predicted by the Coulomb failure model (dashed lines in Figure 10). The assumptions made in the Coulomb model include fault shear strength of 2.2 $\mathrm{MPa}$ and a stress drop of 0.6 $\mathrm{MPa}$ when the failure threshold is reached. Both of these values are averages determined from our data (Figure 1). The Coulomb threshold follows a constant stressing rate amplitude because events are triggered more quickly as stressing rate increases and the failure threshold is reached more often. This boundary underestimates the correlation threshold in the low-frequency regime; however, the slope of the boundary is consistent with the slope of the correlation threshold for the data (Figure 10). Figures 10a and 10b show that the threshold is not well matched by the Coulomb model between $\mathrm{f}_{0}$ and $\mathrm{f}_{\mathrm{c}}$. The high-frequency regime does not follow the slope of the Coulomb threshold except perhaps where the high- 

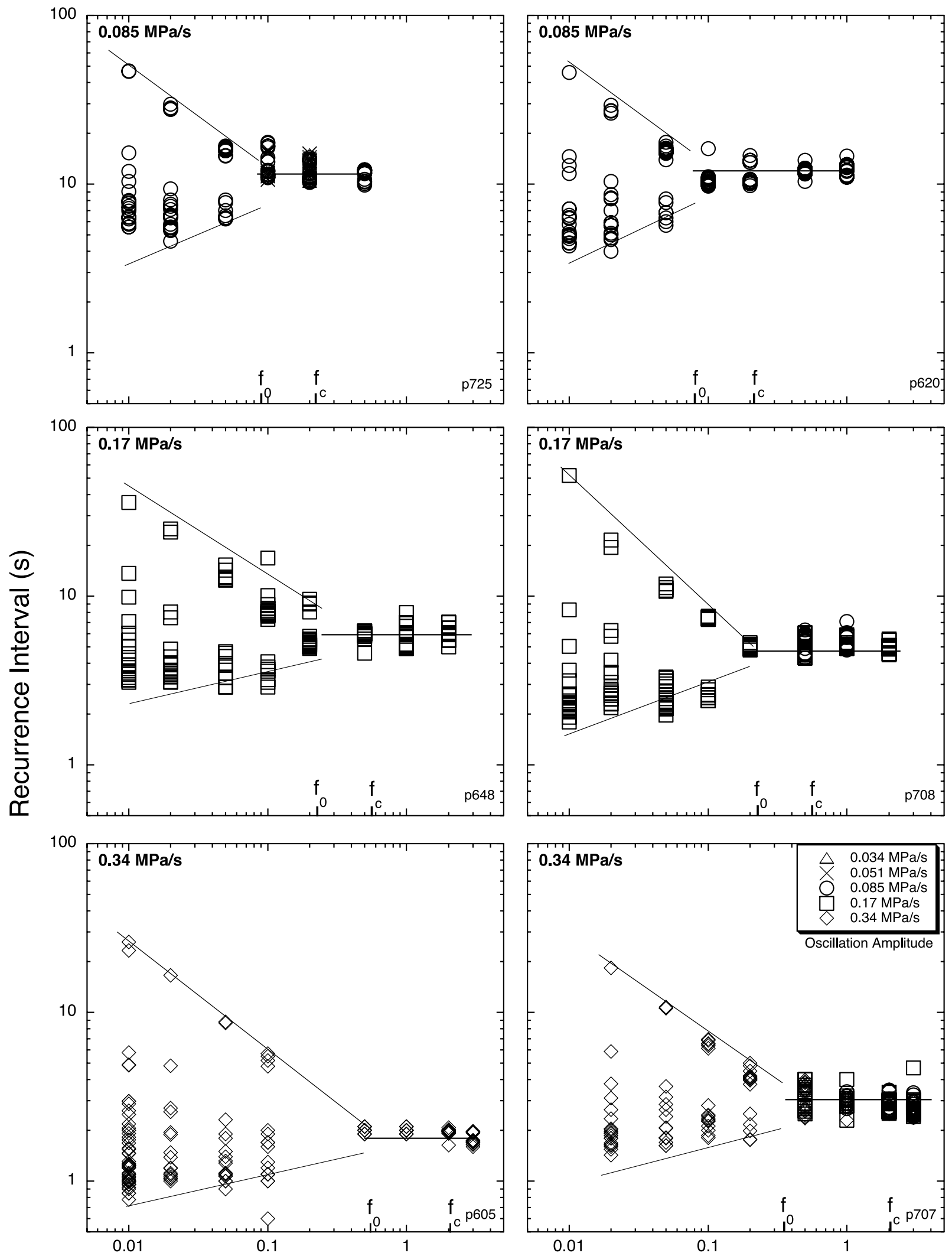

Frequency $(\mathrm{Hz})$

Figure 5. Stick-slip recurrence interval for correlated points in Figure 4. Two experiments are shown for each loading rate to demonstrate repeatability. The frequencies $f_{0}$ and $f_{c}$, which represent the seismicity rate of a nonoscillating experiment and the critical frequency, respectively, are shown for each background loading rate. Solid black lines highlighting trends in recurrence intervals are estimated by eye. 

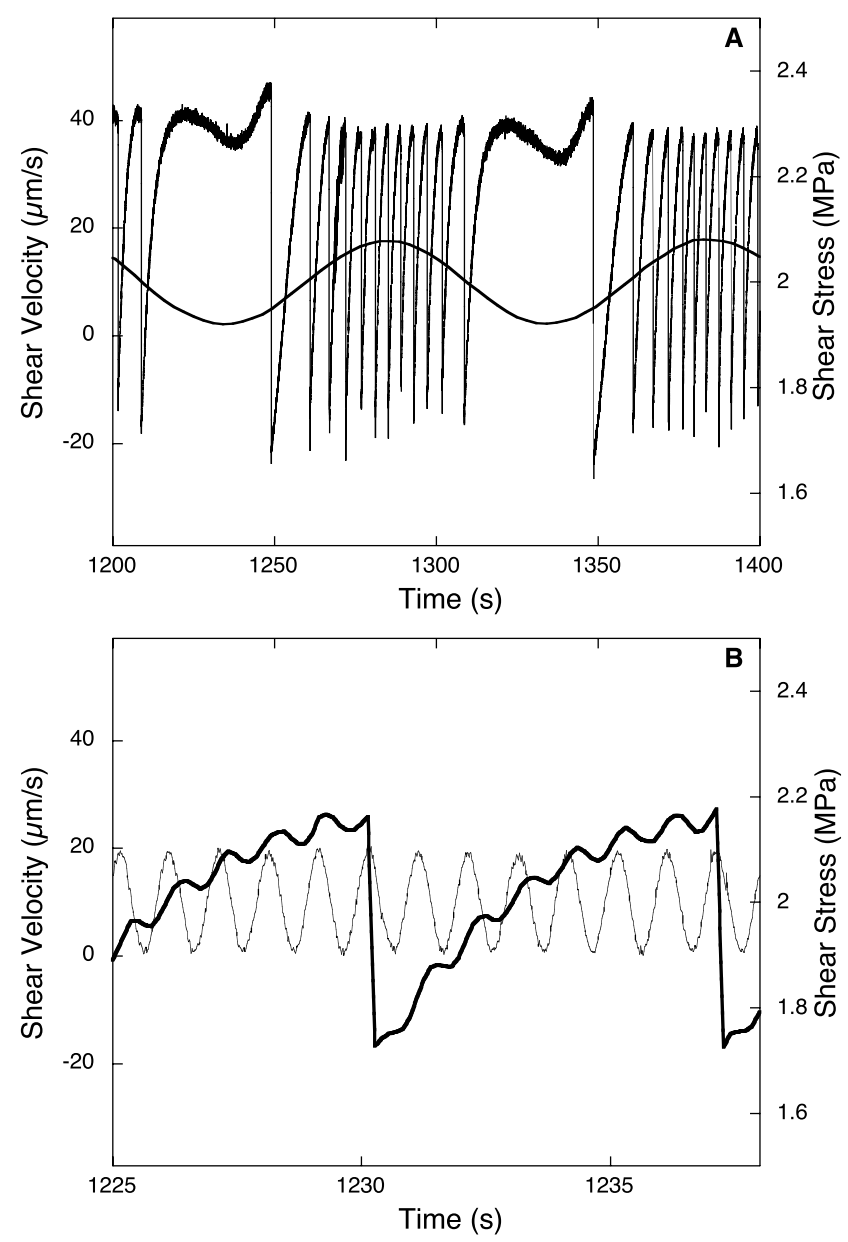

Figure 6. Two examples of correlated response to (a) lowfrequency and (b) high-frequency oscillations. Figure $6 \mathrm{a}$ shows a $0.01 \mathrm{~Hz}$ stressing rate oscillation where most instabilities cluster around the peak stressing rate. Figure 6 shows a $1 \mathrm{~Hz}$ stressing rate oscillation where shear instabilities occur after the stressing rate maximum.

frequency threshold is not well established (Figure 10c). Figures 10a and 10b show that the high-frequency threshold requires larger stress amplitudes than predicted by the Coulomb model. In the high-frequency regime, where the frequency-independent threshold is not well established, the data still show breaks in the slope that are inconsistent with a linear model such as predicted by the Coulomb law.

[25] To explore the possible implications of our observations for triggering by dynamic stresses, we make use of Dieterich's theory of seismicity rate changes after an earthquake [Dieterich, 1994]. Dieterich [1987, 1994] proposed that the increase in seismicity rates following an earthquake generally decays to background seismicity rates over a relaxation time, $\mathrm{t}_{\mathrm{a}}$, determined by

$$
t_{a}=\frac{a \sigma}{\tau_{r}}
$$

where $\sigma$ is normal stress on the fault, $\tau_{\mathrm{r}}$ is the reference shear stressing rate and $a$ is the rate state friction parameter, which is proportional to the direct change in friction following a step change in loading rate. Subsequent studies have suggested the relaxation time is analogous to the inverse of the critical frequency [Beeler and Lockner, 2003]. The friction parameter $a$ for our experiments, obtained through an iterative, least squares inversion of the frictional response to a velocity step, was determined to be 0.008 for glass beads. This inversion was performed on several velocity steps in an experiment run at low normal stress to create stable sliding. Using the laboratory-derived value of $a$, equation (3) predicts critical frequencies of 2.1, 4.3, $8.5 \mathrm{~Hz}$ for the three loading rates used. These values overestimate the observed critical frequencies at all loading rates (Figure 10).

[26] Dieterich's formulation of seismicity rate change simplifies laboratory rate and state friction laws [Dieterich, 1979]. In the seismicity rate formulation, friction evolution is not dependent on the critical slip distance, whereas in the full rate and state friction relations, the steady state state variable (average contact lifetime) is determined by slip velocity and the critical slip distance. We propose that the steady state state variable may be analogous to the inverse of the critical frequency so that

$$
f_{c}=\frac{V_{l p}}{D_{c}}
$$

where $\mathrm{V}_{\mathrm{lp}}$ is the load point velocity (equal to slip velocity at steady state) and $D_{c}$ is the critical displacement needed for friction evolution according to rate and state friction laws. The value of $\mathrm{D}_{\mathrm{c}}$ for glass beads was also calculated by an inversion of the frictional response to a velocity step and was found to be $12 \mu \mathrm{m}$, which is similar to critical friction distances for granular materials [Mair and Marone, 1999]. Equation (4) is similar to the inverse of the critical period discussed by Perfettini and Schmittbuhl [2001] and predicts critical frequencies of $0.4,0.83$, and $1.67 \mathrm{~Hz}$ for stressing rates of $0.085,0.17$, and $0.34 \mathrm{MPa} / \mathrm{s}$, respectively (Figure 10). Although some uncertainty must be attached to the determination of our critical frequencies, the values predicted by equation (4) are always closer to those measured in the lab than those predicted by equation (3).

\subsection{Change in Recurrence Intervals and Phase Lag With Frequency}

[27] The dependence of recurrence interval and phase lag on the frequency of stressing rate variations is a potentially measurable quantity with seismic data. We model the recurrence and phase lag of stick slips using both the Coulomb failure law and the Dieterich rate and state friction law [e.g., Marone, 1998]:

$$
\begin{gathered}
\mu=\mu_{0}+a \ln \left(\frac{V}{V_{0}}\right)+b \ln \left(\frac{V_{0} \theta}{D_{c}}\right) \\
\frac{d \theta}{d t}=1-\frac{V \theta}{D_{c}}
\end{gathered}
$$

where $\mu_{0}$ is a reference friction value, $V_{0}$ is a reference sliding velocity, $\mathrm{V}$ is the fault slip rate, $\mathrm{D}_{\mathrm{c}}$ is the critical slip 


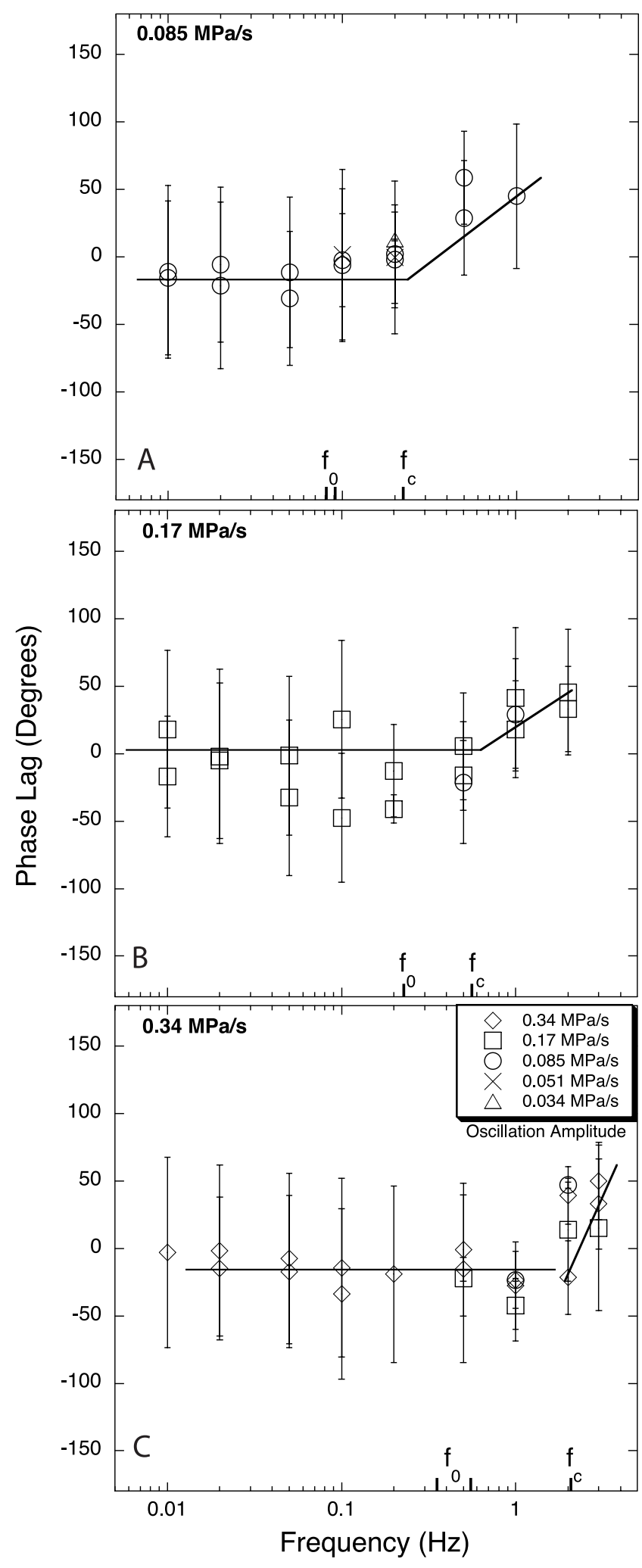

Figure 7. Instability phase shift for correlated points in Figure 4. As in Figure 5, two experiments are plotted for each background loading rate. The mean phase shift for all stick slips at a given frequency is plotted with error bars representing \pm one standard deviation. A positive shift in phase is seen at $f_{c}$. Solid black lines highlighting trends in recurrence intervals are estimated by eye. distance, $\theta$ is a state variable, and $a$ and $b$ are empirically derived constants.

[28] We ran a simple spring-slider block forward model using the parameters described above, a value of 0.05 for the friction parameter $b$ and a stiffness of $0.0175 \mathrm{MPa} / \mu \mathrm{m}$. We describe the elastic interaction between our sample and testing apparatus as

$$
\frac{d \mu}{d t}=k\left(V_{l p}-V\right)
$$

where $\mathrm{k}$ is stiffness divided by normal stress. In the rate and state model, we determine slip to be unstable when the sliding velocity exceeds $110 \%$ of the load point velocity, whereas the Coulomb model fails when shear stress levels exceed a constant shear strength threshold. As in our experiments, we define recurrence interval as the time between the beginning of one instability and the beginning of the next. Phase lag is the phase of the stress oscillation when instability occurs, with the maximum stressing rate at zero phase.

[29] Both models mimic the recurrence rates seen in our experiments (Figure 11). This result is not surprising at low frequencies where the changes in the stress perturbations are slow enough that surfaces have time to evolve to a new friction level before loading rate decreases. The two laws differ at high frequencies where evolution of frictional strength lags the changes in stressing rate, thereby damping the response to the oscillations (and creating the frequencyindependent threshold at high-frequency seen in our experiments). However, in some cases, the resultant change in recurrence time is small enough that the two laws become indistinguishable from one another and from the average recurrence for a nonoscillating system (Figure 11).

[30] We also compare phase lags predicted by a Coulomb failure law and a rate and state friction law to our experimental data (Figure 12). The Coulomb model yields a poor fit to the data near the maximum stressing rate (zero phase) at all frequencies. The rate and state friction law better fits our data, which shows a positive shift in phase lag at frequencies higher than $\mathrm{f}_{\mathrm{c}}$. However, this positive phase shift is only approximately $30^{\circ}$ rather than the $90^{\circ}$ phase shift reported in previous experiments [Beeler and Lockner, 2003] and studies of seismicity and solid Earth tides [Cochran et al., 2004].

\subsection{Granular Force Chains}

[31] Although we do not observe force chains directly, their importance in granular mechanics is sufficiently well established that we propose the following interpretation of our results. We posit that force chain mechanics coupled with adhesive frictional contact mechanics produce three regimes in terms of loading oscillation frequency. The highfrequency regime $\left(f>f_{c}\right.$ ) is defined in terms of the correlation threshold as described above. The low-frequency regime $\left(f<f_{0}\right)$ is bounded by $f_{0}$, which represents the inverse of recurrence time between stick-slip events in a nonoscillating system. This is the length of time needed to create and subsequently break force chains at a given background loading rate. Previous work shows that recurrence time increases with shear layer thickness and implies that $1 / f_{0}$ increases with the number of particles in a force 


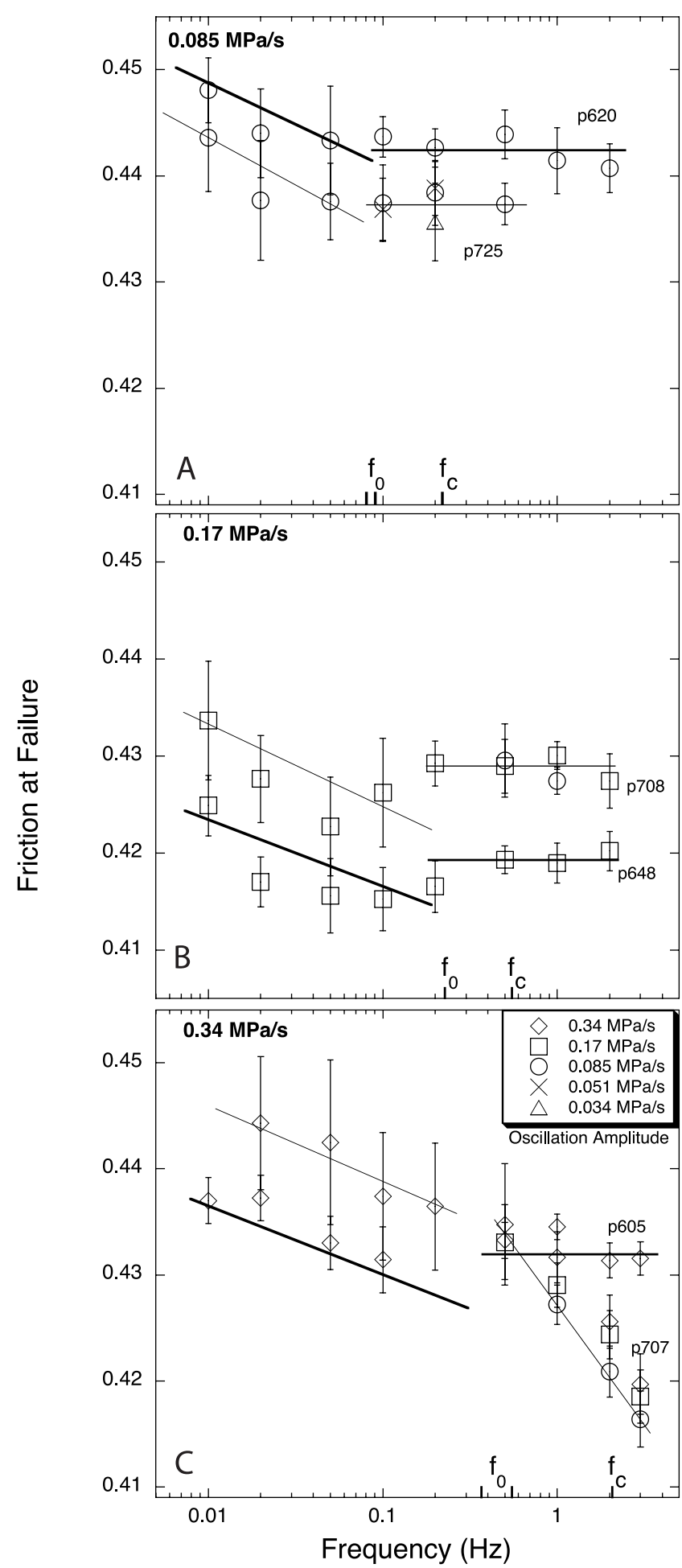

Figure 8. Friction at failure for correlated points in Figure 4. Failure strength decreases with frequency for $F<$ $\mathrm{f}_{0}$ for each background loading rate. Above $\mathrm{f}_{0}$, frictional failure values are constant or in one case decrease (p707). Frictional strength is independent of loading rate oscillation amplitude for the range of conditions studied. Error bars represent one standard deviation. Solid black lines highlighting trends in recurrence intervals are estimated by eye. chain [Anthony and Marone, 2005]. We propose that at oscillation frequencies lower than $\mathrm{f}_{0}$, force chains form, buckle, and break much the same way they would in a nonoscillating system; however, the failure strength decreases with increasing frequency. If we consider a given stick-slip cycle, our hypothesis is that shear load oscillations bend force chains, but only transiently, because shearing ceases before chains fail. However, successive oscillations progressively weaken the chains, such that failure occurs at a lower friction level compared to nonoscillating systems, as we observe (Figure 8). This weakening in failure strength may be reflected in the fact that the correlation threshold at low frequencies has a greater negative slope than the Coulomb failure law predicts. At frequencies higher than $\mathrm{f}_{0}$ the oscillation period is shorter than the time needed to shear a force chain by the effective critical slip distance for a granular layer, which is proportional to the contact critical slip distance scaled by the number of contacts in the chain [Marone and Kilgore, 1993; Marone, 1998]. This indicates that incremental contact weakening by shear oscillations, which at lower frequencies was effective in disrupting adhesive frictional aging, becomes negligible, and failure strength becomes independent of frequency, as our data for the two lower background shearing rates show (Figure 8). The effect is clearest at the intermediate background shearing rate. At the highest background loading rate, the $0.34 \mathrm{MPa} / \mathrm{s}$ oscillations show a tendency to flatten out in the range $0.2-1 \mathrm{~Hz}$; however, yield strength continues to decrease for higher oscillation frequencies in one experiment. This may be related to larger-scale particle rearrangement and effects that are not represented by rate/ state friction theory.

[32] Our model also supports an explanation for the preseismic slip data (Figure 9). For frequencies below $f_{0}$, oscillation-induced disruption of contact aging causes increasing inelastic (preseismic) slip with increasing frequency. This is consistent with a model in which creep accumulates with each load oscillation and force chains fail via progressive creep prior to unstable buckling. The trend of increasing preseismic creep with increasing frequency up to $f_{0}$ is observed at each background loading rate and indicates that force chains are made less brittle by loading oscillations (Figure 9). For frequencies above $f_{0}$, preseismic slip becomes constant for our two lowest loading rates, consistent with the observation of constant yield stress in this regime. For our highest background loading rate, preseismic slip decreases for $f>\mathrm{f}_{0}$. This observation may be related to the continued reduction in yield stress in that case. The positive correlation between preseismic slip and coseismic slip is consistent with the work of Anthony and Marone [2005]. In summary, the two important frequencies in our model, $\mathrm{f}_{\mathrm{c}}$ and $\mathrm{f}_{0}$, reflect the time necessary to slip the frictional critical slip distance for a single contact junction and that which corresponds to the force chain as a whole.

\subsection{Tectonic Implications}

[33] The important parameters that need to be scaled up to tectonic faults are the critical frequency and the critical displacement length. Our equation for critical frequency (equation (4)) implies that the nucleation time is approximately 0.3 years for a fault with a tectonic plate rate of $30 \mathrm{~mm} / \mathrm{yr}$ (such as the San Andreas) and critical displace- 

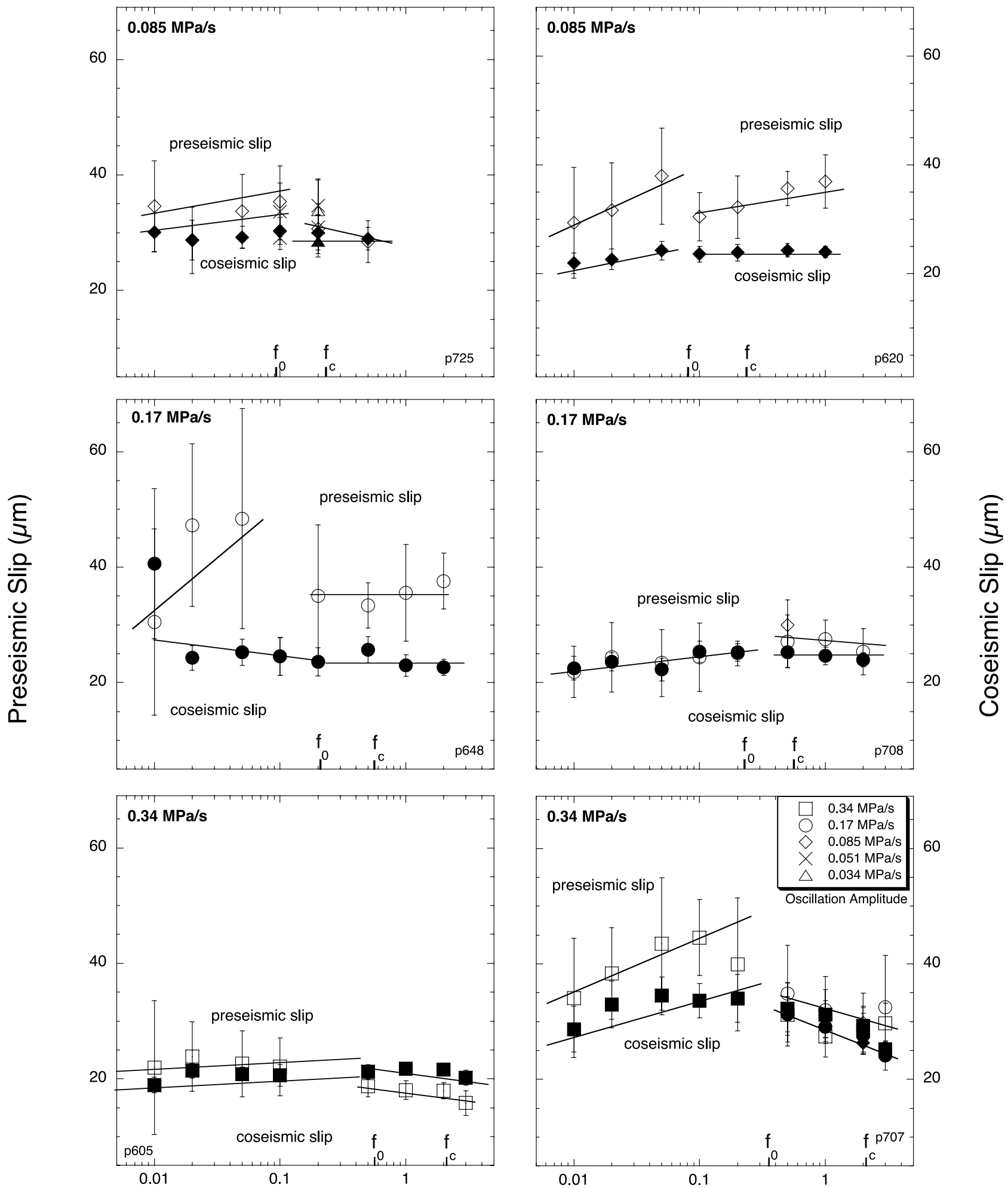

\section{Frequency $(\mathrm{Hz})$}

Figure 9. Changes in creep and coseismic slip with varying frequency. Creep is shown with open symbols whereas coseismic slip is represented with solid symbols. Each row represents a background loading rate (with two experiments in each row to demonstrate repeatability. Preseismic slip and coseismic slip tend to covary, but the variation in displacement for coseismic slip is smaller. Solid black lines highlighting trends in recurrence intervals are estimated by eye. 

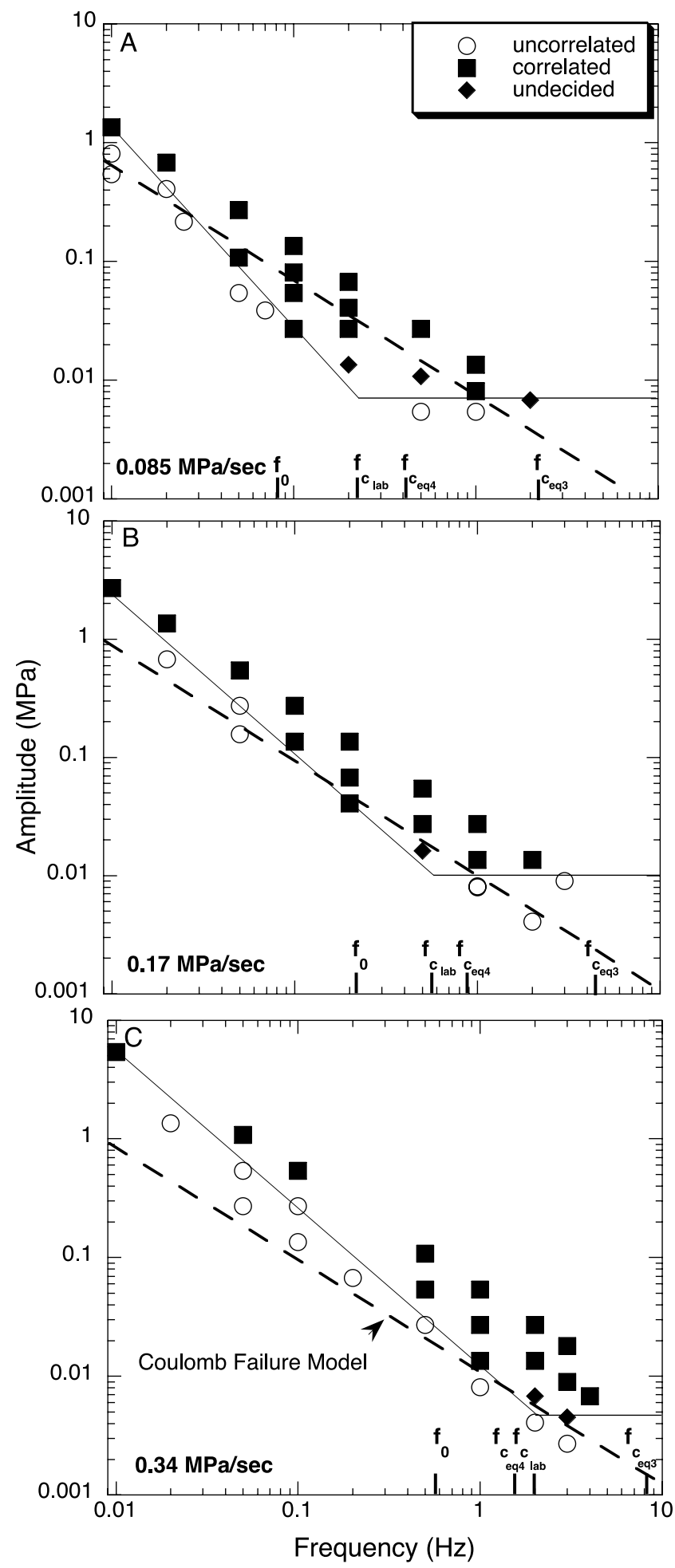

Figure 10. Correlation data shown in Figure 4 with correlation threshold predicted the Coulomb failure model (dashed line) and critical frequencies predicted by equations (3) and (4).

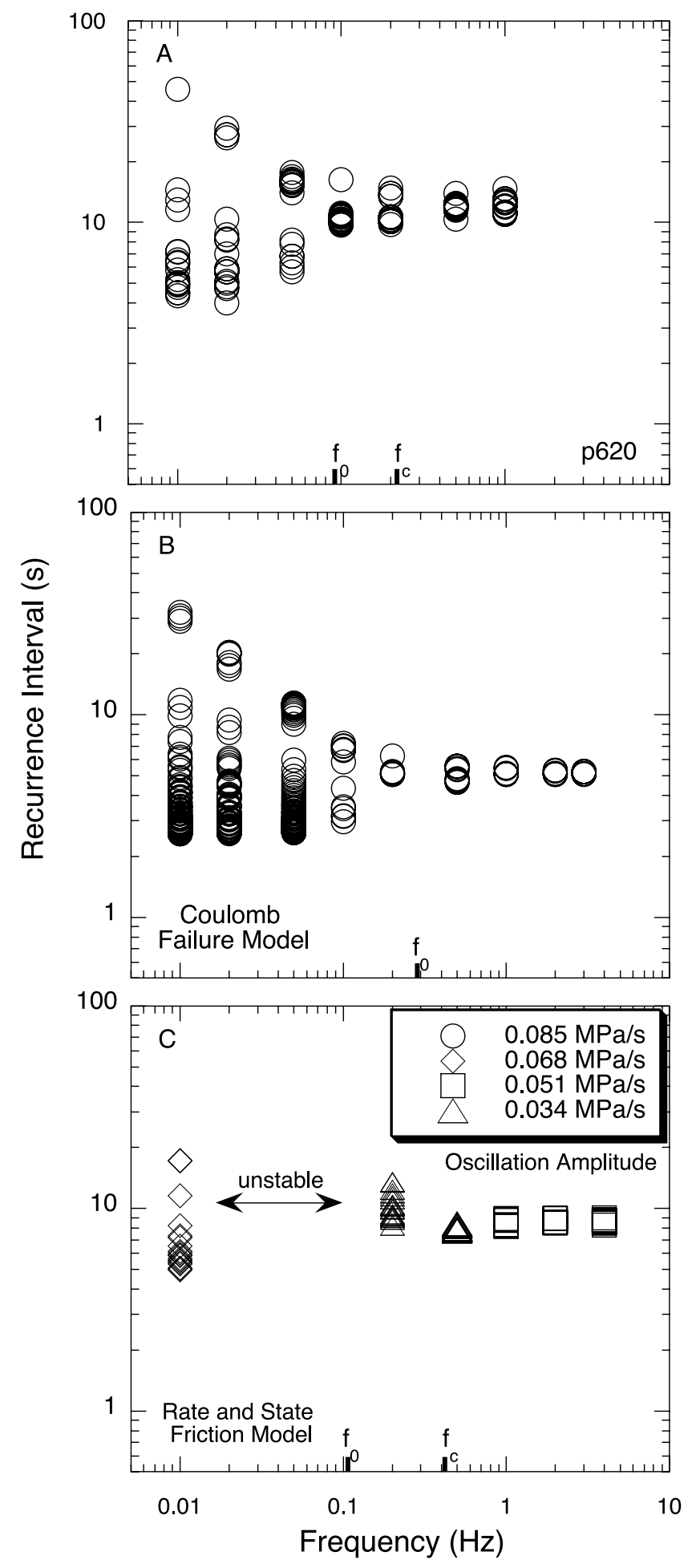

Figure 11. Dependence of recurrence intervals on stressing rate frequency from (a) the laboratory and predicted by (b) the Coulomb failure criterion and (c) the rate and state friction equations. The gap in low-frequency data points in Figure $11 \mathrm{c}$ represents conditions in which the model becomes unstable. Stressing rate is $0.085 \mathrm{MPa} / \mathrm{s}$ in all plots. All points on the plot represent amplitude and frequency combinations that produce failure times correlating with the oscillating stress with $95 \%$ confidence. 


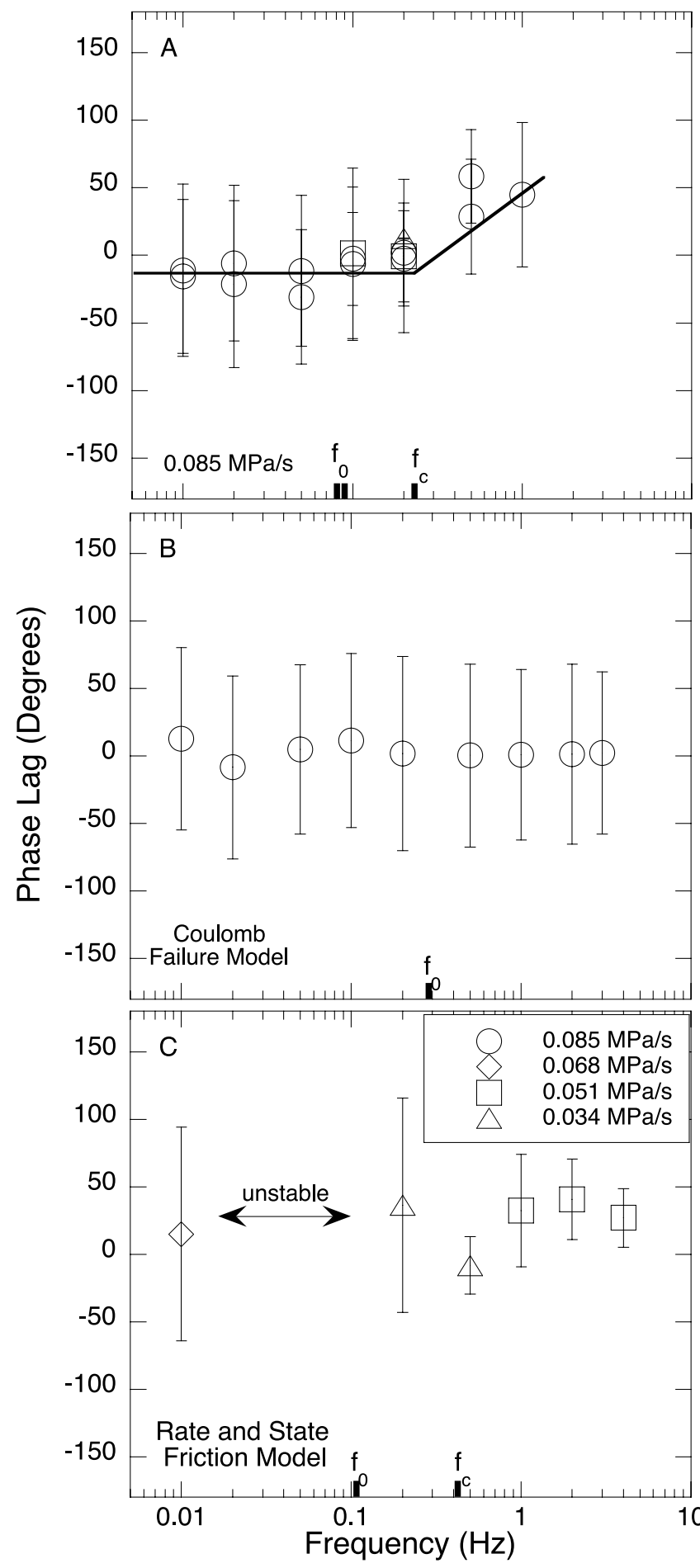

Figure 12. Dependence of phase lag on stressing rate frequency from (a) the laboratory and predicted by (b) the Coulomb failure criterion and (c) the rate and state friction equations. All points in Figures $12 \mathrm{~b}$ and $12 \mathrm{c}$ represent the same model conditions as those in Figure 11.

ment length of $10 \mathrm{~mm}$. Previous calculations have estimated the crossover time for tectonic faults to be in the range of approximately 1 year [Toda et al., 2002] to 18.2 years [Beeler and Lockner, 2003]. This implies that seasonal loading, Earth tidal stresses and earthquake waves would be in the high-frequency regime. Timing of failure in this regime is controlled by rate and state friction, i.e., a small nondynamic displacement must occur before unstable slip occurs, explaining why unstable slip is delayed relative to the maximum stressing rate. Plots of earthquake occurrence over the tidal period show that the maximum number of events occurs at the peak tidal stress [Cochran et al., 2004], as predicted by rate and state laws. Toda et al. [2002] suggested that the stressing rate near a tectonically active area (e.g., dike intrusions) would have a shorter crossover time (on the order of hours) which would make the tidal frequency in active seismic areas near the critical frequency or in the low-frequency regime. We should note that our shear velocity experiments do not simulate a seismic wave but represents the small shear velocity that would be resolved across a fault as the wave impinged and caused different displacement rates on different sides of the fault.

[34] The critical displacement $D_{c}$ depends not only on asperity contacts but also on gouge zone width and/or shear localization width and thus will vary with net fault offset and shear strain. Laboratory experiments have shown that the critical displacement decreases with an increase in shear strain as strain localizes into thinner bands of deformation [Marone and Kilgore, 1993]. This implies that faults with a thick gouge layer and low shear strain would have larger $D_{c}$ and therefore a lower critical frequency than faults with thinner gouge layers and less shear strain. Additionally, the correlation threshold for tectonic faults may depend on the degree of gouge formation and the interaction of grains within that gouge layer. Faults lacking a gouge layer or with a highly developed gouge layer (with discrete shear bands) may be less sensitive to high-frequency oscillations because of the absence or diffusiveness of force chains. However, in less consolidated material, high-frequency oscillations should trigger earthquakes at smaller stress amplitudes than expected.

\section{Conclusions}

[35] Shear loading rate experiments show that amplitude and frequency of shear stress oscillations determine whether instabilities are triggered. Two regimes of failure are documented: a low-frequency regime where the correlation condition depends on both amplitude and frequency of the shear oscillation and a high-frequency regime where the correlation condition was frequency-independent. The phase lag of failure with respect to the stressing rate curve, the frictional strength and the recurrence time between events are all influenced by frequency of the oscillation. Some aspects of our data, such as recurrence patterns, can be explained by simple failure laws, whereas aspects such as phase lag suggest more complicated behavior. Two important frequencies in determining changes in failure characteristics are the inverse of the time needed to break force chains and the time needed to renew grain-grain contacts.

[36] Acknowledgments. This manuscript benefited greatly from reviews by Joan Gomberg, Paul Johnson, and Emily Brodsky. This work was supported by National Science Foundation grants EAR 0196570, OCE 0196462, EAR-0337627, and EAR-0345813 and by USGS/NEHRP award 05HQGR0025. 


\section{References}

Anthony, J., and C. Marone (2005), Influence of particle characteristics on granular friction, J. Geophys. Res., 110, B08409, doi:10.1029/ 2004JB003399.

Beeler, N. M., and D. A. Lockner (2003), Why earthquakes correlate weakly with the solid Earth tides: Effects of periodic probability of earthquake occurrence, J. Geophys. Res., 108(B8), 2391, doi:10.1029/ 2001JB001518.

Bindschadler, R., M. King, R. Alley, S. Anandakrishnan, and L. Padman (2003), Tidally controlled stick-slip discharge of a West Antarctic ice stream, Science, 301, 1087-1089.

Boettcher, M. S., and C. Marone (2004), Effects of normal stress variations on the strength and stability of creeping faults, J. Geophys. Res., 109 , B03406, doi:10.1029/2003JB002824.

Brodsky, E. E., and S. G. Prejean (2005), New constraints on mechanisms of remotely triggered seismicity at Long Valley Caldera, J. Geophys. Res., 110, B04302, doi:10.1029/2004JB003211.

Brodsky, E. E., E. Roeloffs, D. Woodcock, I. Gall, and M. Manga (2003), A mechanism for sustained groundwater pressure changes induced by distant earthquakes, J. Geophys. Res., 108(B8), 2390, doi:10.1029/ 2002JB002321.

Cochard, A., L. Bureau, and T. Baumberger (2003), Stabilization of frictional sliding by normal load modulation, J. Appl. Mech., 70, 220-226.

Cochran, E. S., J. E. Vidale, and T. Sachiko (2004), Erth tides can trigger shallow thrust fault earthquakes, Science, 306, 1164-1166.

Dieterich, J. (1979), Modeling of rock friction: 1. Experimental results and constitutive equations, J. Geophys. Res., 84, 2161-2168.

Dieterich, J. (1987), Nucleation and triggering of earthquake slip; effects of periodic stress, Tectonophysics, 144, 127-139.

Dieterich, J. (1994), A constitutive law for rate of earthquake production and its application to earthquake clustering, J. Geophys. Res., 99, 26012618.

Felzer, K., and E. Brodsky (2006), Decay of aftershock density with distance indicates triggering by dynamic stress, Nature, 441, 735-738.

Frye, K., and C. Marone (2002), Effect of humidity on granular friction at room temperature, J. Geophys. Res., 107(B11), 2309, doi:10.1029/ 2001JB000654.

Gomberg, J., and P. A. Johnson (2005), Seismology: Dynamic triggering of earthquakes, Nature, 437, 830, doi:10.1038/437830a

Gomberg, J., M. Blanpied, and N. M. Beeler (1997), Transient triggering of near and distant earthquakes, Bull. Seismol. Soc. Am., 87, 294-309.
Gomberg, J., P. Bodin, K. Larson, and H. Dragert (2004), Earthquake nucleation by transient deformations caused by the $M=7.9$ Denali, Alaska earthquake, Nature, 427, 621-624.

Heki, K. (2003), Snow load and seasonal variation of earthquake occurrence in Japan, Earth Planet. Sci. Lett., 20, 159-164.

Hong, T., and C. Marone (2005), Effect of normal stress perturbations on the frictional properties of simulated faults, Geochem. Geophys. Geosyst. 6, Q03012, doi:10.1029/2004GC000821.

Johnson, P., and X. Jia (2005), Nonlinear dynamics, granular media and dynamic earthquake triggering, Nature, 437, 871-874.

Lammlein, D. R. (1977), Lunar seismicity, structure, and tectonics, Philos. Trans. R. Soc. London, Ser. A, 285, 451-461.

Lockner, D. A., and N. M. Beeler (1999), Premonitory slip and tidal triggering of earthquakes, J. Geophys. Res., 104, 20,133-20,151.

Mair, K., and C. Marone (1999), Friction of simulated fault gouge for a wide range of velocities and normal stresses, J. Geophys. Res., 104 28,899-28,914.

Marone, C. (1998), Laboratory-derived friction laws and their application to seismic faulting, Annu. Rev. Earth Planet. Sci., 26, 643-696.

Marone, C., and B. Kilgore (1993), Scaling of the critical slip distance for seismic faulting with shear strain in fault zones, Nature, 362, 618-621.

Perfettini, H., and J. Schmittbuhl (2001), Periodic loading on a creeping fault: Implications for tides, Geophys. Res. Lett., 28, 435-438.

Richardson, E., and C. Marone (1999), Effects of normal stress vibrations on frictional healing, J. Geophys. Res., 104, 28,859-28,878.

Rydelek, P., and L. Hass (1994), On estimating the amount of blasts in seismic catalogs with Schuster's method, Bull. Seismol. Soc. Am., 84 $1256-1259$

Saar, M., and M. Manga (2003), Seismicity induced by seasonal groundwater recharge at Mt. Hood, Oregon, Earth Planet. Sci. Lett., 214, 605618

Toda, S., R. Stein, and T. Sagiya (2002), Evidence from the AD 2000 Izu islands earthquake swarm that stressing rate governs seismicity, Nature, 419, 58-61.

Tolstoy, M., F. Vernon, J. Orcutt, and F. Wyatt (2002), Breathing of the seafloor; tidal correlations of seismicity at Axial volcano, Geology, 30 , $503-506$.

C. Marone and H. M. Savage, Rock Mechanics Laboratory, Department of Geosciences, Pennsylvania State University, University Park, PA 16802, USA. (cjm@geosc.psu.edu; hsavage@geosc.psu.edu) 